\title{
Modeling of realistic cladding structures for air-core photonic band-gap fibers
}

\author{
Niels Asger Mortensen \\ Crystal Fibre A/S, Blokken 84, DK-3460 Birkerød, Denmark \\ Martin Dybendal Nielsen \\ Crystal Fibre A/S, Blokken 84, DK-3460 Birkerød, Denmark \\ COM, Technical University of Denmark, DK-2800 Kongens Lyngby, Denmark

\begin{abstract}
Cladding structures of photonic band-gap fibers often have air-holes of noncircular shape and, typically, close-to-hexagonal air holes with curved corners are observed. We study photonic band-gaps in such structures by aid of a twoparameter representation of the size and curvature. For the fundamental bandgap we find that the band-gap edges (the intersections with the air line) shift toward shorter wavelengths when the air-filling fraction $f$ is increased. The band-gap also broadens and the relative band-width increases exponentially with $f^{2}$. Comparing to recent experiments [Nature 424, 657 (2003)] we find very good agreement.
\end{abstract}

(C) 2018 Optical Society of America

OCIS codes: $060.2280,060.0060$

In air-silica photonic crystal fibers (PCFs) an arrangement of air-holes running along the full length of the fiber provides the confinement and guidance of light in a defect region. For photonic band-gap (PBG) guiding PCFs the air-holes have been arranged in various ways such as in a triangular lattice ${ }^{\underline{1}}$, but honey-comb ${ }^{2}$ and kagome $e^{3}$ arrangements are other options. Cregan et al. . $^{\frac{1}{1}}$ have demonstrated that light can be guided in an aircore by means of the PBG effect and this observation has stimulated an avalanche of both basic and applied research. For recent reviews we refer to Refs. 4,5 and references therein.

From a modeling point of view the air-holes are generally assumed circular ${ }^{6.7}$ and for fibers fabricated by the stack-and-pull method this is also typically what is observed for moderate air-filling fractions $\stackrel{\underline{8}}{=}$ However, for the high air-filling fractions $f>0.8$ recently employed for PBG guidance the air-holes tend to be noncircular ${ }^{9,10}$ From a simple geometric consideration it is

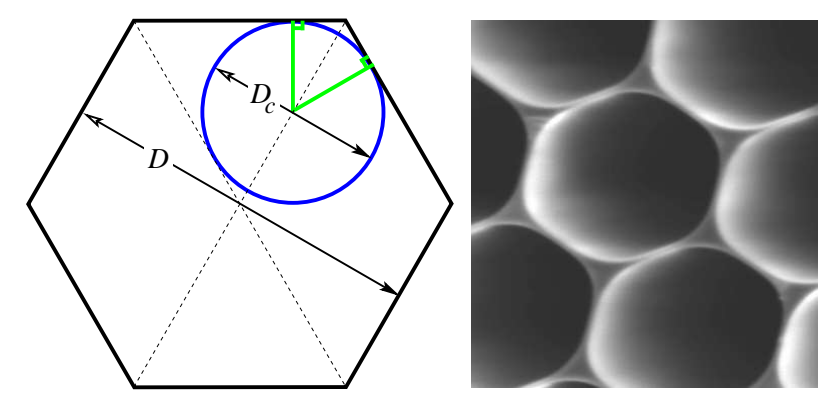

Fig. 1. The right panel shows a scanning-electron micrograph of the cladding structure of a PBG fiber with a pitch of $\Lambda \simeq 2.7 \mu \mathrm{m}$. The left panel illustrates the two-parameter representation of the air-hole shape. easily shown that the theoretical upper limit of the airfilling fraction in a structure of close packed circular voids is $f=\pi /(2 \sqrt{3}) \simeq 0.91$. Hence, design and fabrication of air-core PBG fibers call for a detailed modeling of the spectral position of the PBGs which goes beyond the assumption of circular air holes. In this Letter we consider the triangular cladding arrangement (with a pitch $\Lambda$ ) first employed by Cregan et al.,$\frac{1}{\underline{1}}$ but for the air-holes we take the non-circular shape observed recently in Refs. 9 , 10 into account. From scanning-electron micrographs (SEMs) we find that the air-holes are of over-all hexagonal shape with curved corners, see Fig. 1 (this conclusion was also emphasized in Ref. 10). This suggests that the holes can be parametrized by two parameters; the edge-to-edge distance $D$ (corresponding to the diameter of a circular airhole) and the diameter of curvature, $D_{c}$, at the corners (see left panel of Fig. (1). In this two-parameter representation the air-filling fraction is given by

$$
\begin{aligned}
f & =\frac{A_{\mathrm{hex}}(D)-A_{\mathrm{hex}}\left(D_{c}\right)+A_{\mathrm{circ}}\left(D_{c}\right)}{A_{\mathrm{hex}}(\Lambda)} \\
& =\left(\frac{D}{\Lambda}\right)^{2}\left[1-\left(1-\frac{\pi}{2 \sqrt{3}}\right)\left(\frac{D_{c}}{D}\right)^{2}\right]
\end{aligned}
$$

where $A_{\text {hex }}(x)=\sqrt{3} x^{2} / 2$ and $A_{\text {circ }}(x)=\pi(x / 2)^{2}$ are the areas of a hexagon (with edge-to-edge distance $\mathrm{x}$ ) and a circle (of diameter $x$ ), respectively.

For the optical properties we apply Maxwell's equations to a macroscopic and isotropic loss-less dielectric medium and assume a linear dependence of the displacement field on the electrical field. We consider a harmonic mode $\boldsymbol{H}(\boldsymbol{r}, t)=\boldsymbol{H}_{\omega}(\boldsymbol{r}) e^{i \omega t}$ with angular frequency $\omega$ and substituting into Maxwell's equations the magnetic-field 


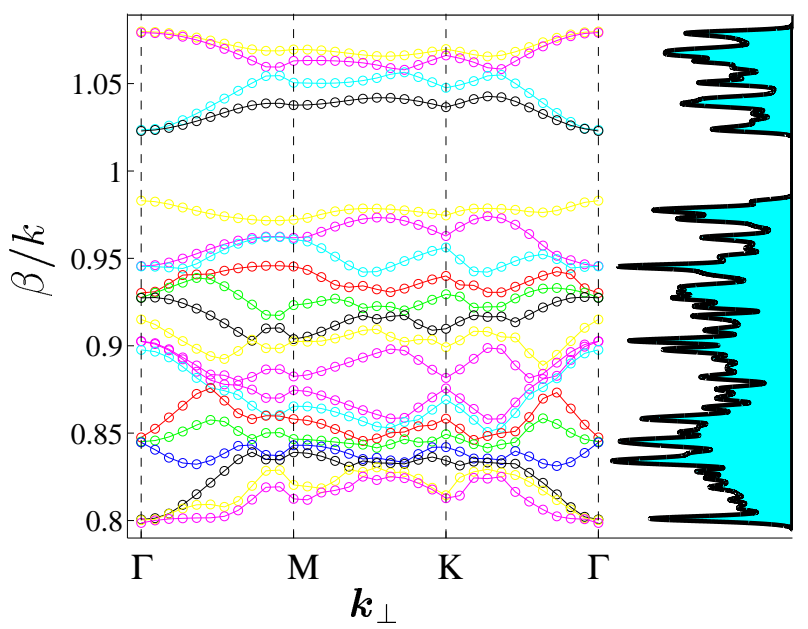

Fig. 2. Plot of $\beta / k$ versus $\boldsymbol{k}_{\perp}$ along principal directions in the Brillouin zone for $\beta \Lambda=14.45$ and a structure with $D / \Lambda=0.96$ and $D_{c} / \Lambda=0.55$ (see inset of Fig. 31). The filled curve shows the corresponding densityof-states (the projection of the data onto the $y$-axis).

vector is then governed by the wave equation 11

$$
\boldsymbol{\nabla} \times \frac{1}{\varepsilon(\boldsymbol{r})} \boldsymbol{\nabla} \times \boldsymbol{H}_{\omega}(\boldsymbol{r})=\frac{\omega^{2}}{c^{2}} \boldsymbol{H}_{\omega}(\boldsymbol{r}) .
$$

Here, $c$ is the velocity of light and $\varepsilon$ is the dielectric function which we in the following assume independent of frequency by which the wave equation becomes scaleinvariant. All the results to be presented can thus be scaled to the desired value of $\Lambda$.

For a PCF geometry, the cladding dielectric function $\varepsilon(\boldsymbol{r})$ is periodic in the transverse plane and translational invariant along the fiber-axis (the $z$-axis). The solution is according to Bloch's theorem a plane wave modulated by a function $\boldsymbol{h}_{\omega}\left(\boldsymbol{r}_{\perp}\right)$ with the periodicity of the dielectric structure in the transverse direction

$$
\boldsymbol{H}_{\omega}(\boldsymbol{r})=\boldsymbol{h}_{\omega}\left(\boldsymbol{r}_{\perp}\right) \exp \left(i \boldsymbol{k}_{\perp} \cdot \boldsymbol{r}_{\perp}+i \beta z\right) .
$$

Substituting this ansatz into Eq.(2) we get an eigenvalue problem for $\omega\left(\boldsymbol{k}_{\perp}, \beta\right)$ which we solve by the aid of a planewave basis (typically $128 \times 128$ plane waves) with periodic boundary conditions 12 For the dielectric function we use $\varepsilon=1$ in air and $\varepsilon=(1.444)^{2}=2.085$ in silica. From a computational point of view our simulations thus follow the same lines as those used for structures with circular holes $\underline{\underline{6}}$

In Fig. 2 we show photonic bands calculated for $\beta \Lambda=$ 14.45 and a structure with $D / \Lambda=0.96$ and $D_{c} / \Lambda=0.55$ (see inset of Fig. 31). It is common to introduce the freespace wave-number $k=2 \pi / \lambda=\omega / c$ and plot the effective index $\beta / k=c \beta / \omega$ rather than the angular frequency $\omega$. The bands are plotted as a function of $\boldsymbol{k}_{\perp}$ along the usual principal directions in the Brillouin zone; from the $\Gamma$-point to the $M$-point and via the $K$-point back to the $\Gamma$-point $\underline{\underline{6}}$ As seen the structure exhibits a band-gap which is particular clear from the filled curve which shows the corresponding density-of-states (the projection of the data onto the $y$-axis). In this example the band-gap is around the index of air $(\beta / k=1)$, but in general the position and width of the band-gaps depend on the propagation constant $\beta$. In Fig. 3 we show the results of changing $\beta$. The data-points result from a sampling of the Brillouin zone along the above mentioned principal directions. The PBGs are those regions with no data-points and the solid lines indicate the corresponding band-gap edges. The dashed line shows the air-line which passes through several band-gaps. Usually the band-gap at the longest wavelength (the lowest frequency) is refereed to as the fundamental band-gap and the other gaps are denoted higher-order band-gaps ${ }^{6}$ The slopes of the band-gaps are relatively large which suggest that e.g. scattering and bending-loss will increase almost abruptly when the wavelength approaches the band-edges.

For PBG fibers with an air-core ${ }^{1.9 .10}$ the band-edges of guided modes will to a good approximation be given by the intersection of the air-line with the cladding bandedges in Fig. 3. For the fundamental band-gap we denote the upper and lower intersections by $\lambda_{u}$ and $\lambda_{l}$, respectively. We have calculated the band edges for various

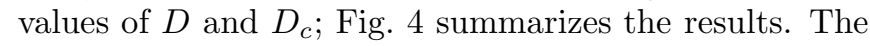
band-diagrams of course depend on the two parameters $D$ and $D_{c}$, but we find that a single-parameter description of the band-edges is possible in terms of the air-filling

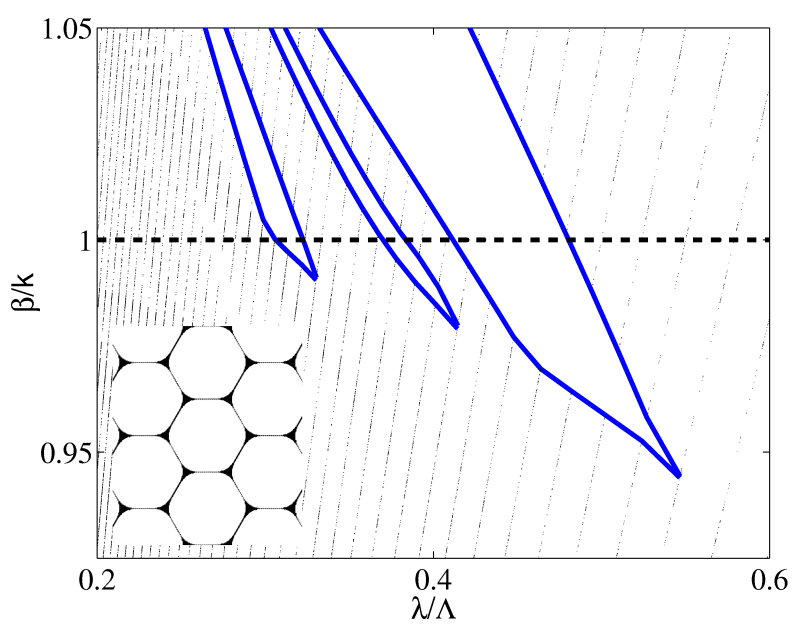

Fig. 3. Plot of $\beta / k$ versus $\lambda / \Lambda$ for a structure with $D / \Lambda=0.96$ and $D_{c} / \Lambda=0.55$ (see inset). The datapoints result from a sampling of the Brillouin zone along the usual path from the $\Gamma$-point to the $M$-point and via the $K$-point back to the $\Gamma$-point. The solid lines indicate the band-gap boundaries and the dashed line $\beta / k=1$ shows the air line which passes through several bandgaps. 


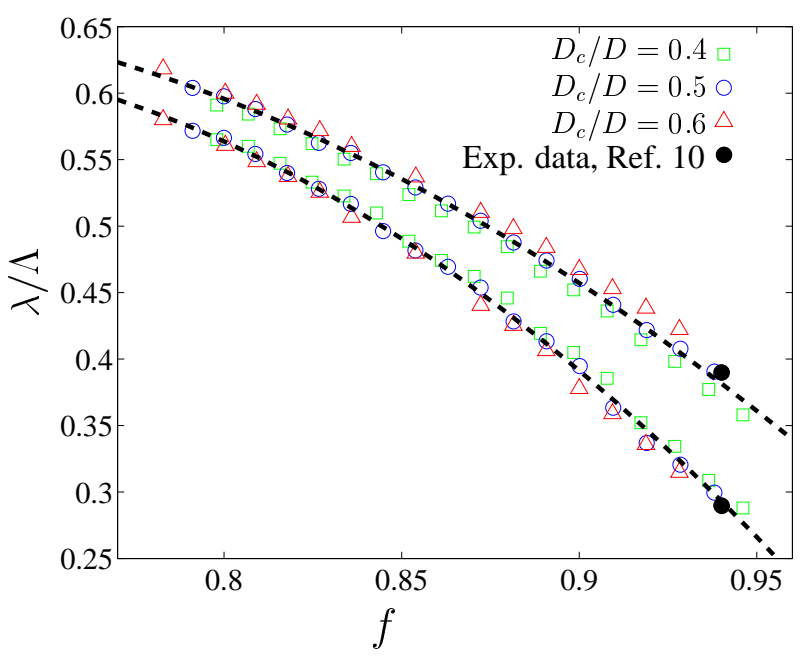

Fig. 4. Plot of the band-gap edges $\lambda_{u}$ and $\lambda_{l}$ of the fundamental band-gap as a function of air-filling fraction $f$. The dashed lines are guides to the eyes. Recent experimental data ${ }^{10}$ is also included.

fraction $f$, Eq. (1).

When the air-filling fraction is increased the center $\lambda_{c}=\left(\lambda_{u}+\lambda_{l}\right) / 2$ of the band-gap shifts toward shorter wavelengths. Furthermore, the band-width $\Delta \lambda=\lambda_{u}-\lambda_{l}$ at the same time increases and the relative band-width shows a close to exponential increase (see Fig. [5), i.e.

$$
\Delta \lambda / \lambda_{c} \sim \mathcal{B} \times \exp \left(\mathcal{A} \times f^{2}\right),
$$

where $\mathcal{A}$ and $\mathcal{B}$ are positive numerical coefficients. The results in Figs. 45 support the choice of high air-filling fractions for practical fibers and as seen we find very good agreement between our numerics and recent experimental results by Smith et al $l^{10}$

In summary, we have shown that realistic PBG cladding structures $\$ .10$ can be represented by an "ideal" two-parameter description which facilitate detailed numerical modeling. For the fundamental band-gap the band-gap edges (the intersections with the air line) shift toward shorter wavelengths for an increasing air-filling fraction $f$ and the band-gap also broadens significantly. This observation may make air-core PBG fibers realistic for wavelengths even shorter than the $850 \mathrm{~nm}$ reported recently by Bouwmans et $a l^{9} \stackrel{9}{n}$

We are grateful to B. H. Larsen (NKT Research) for providing SEMs of real samples and our colleges T. P. Hansen, J. Broeng, and G. Vienne and for stimulating discussions on the experimentally observed structures. M. D. Nielsen acknowledges financial support by the Danish Academy of Technical Sciences. N. A. Mortensen's e-mail address is asger@mailaps.org.

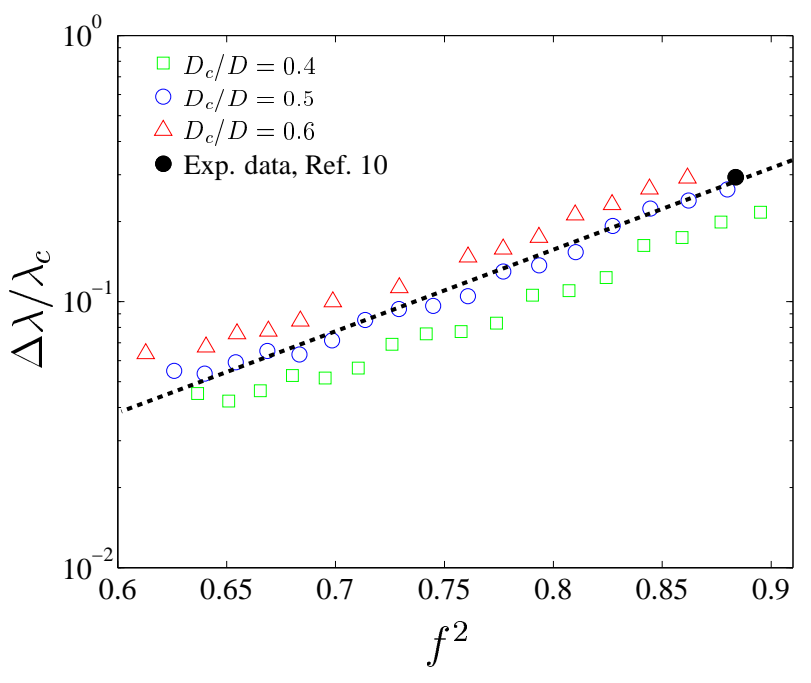

Fig. 5. Relative band-width versus $f^{2}$. The straight dashed line indicates the close-to-exponential dependence, Eq. (4). Recent experimental data 10 is also included.

\section{References}

1. R. F. Cregan, B. J. Mangan, J. C. Knight, T. A. Birks, P. S. J. Russell, P. J. Roberts, and D. C. Allan, Science 285, 1537 (1999).

2. J. C. Knight, J. Broeng, T. A. Birks, and P. S. J. Russell, Science 282, 1476 (1998).

3. F. Benabid, J. C. Knight, G. Antonopoulos, and P. S. J. Russell, Science 298, 399 (2002).

4. P. Russell, Science 299, 358 (2003).

5. J. C. Knight, Nature 424, 847 (2003).

6. J. Broeng, S. E. Barkou, T. Søndergaard, and A. Bjarklev, Opt. Lett. 25, 96 (2000).

7. T. A. Birks, P. J. Roberts, P. S. J. Russell, D. M. Atkin, and T. J. Shephard, Electron. Lett. 31, 1941 (1995).

8. J. C. Knight, T. A. Birks, P. S. J. Russell, and D. M. Atkin, Opt. Lett. 21, 1547 (1996).

9. G. Bouwmans, F. Luan, J. C. Knight, P. S. J. Russel, L. Farr, B. J. Mangan, and H. Sabert, Opt. Express 11, 1613 (2003).

10. C. M. Smith, N. Venkataraman, M. T. Gallagher, D. Müller, J. A. West, N. F. Borrelli, D. C. Allen, and K. W. Koch, Nature 424, 657 (2003).

11. J. D. Joannopoulos, R. D. Meade, and J. N. Winn, Photonic crystals: molding the flow of light (Princeton University Press, Princeton, 1995).

12. S. G. Johnson and J. D. Joannopoulos, Opt. Express 8, 173 (2001). 